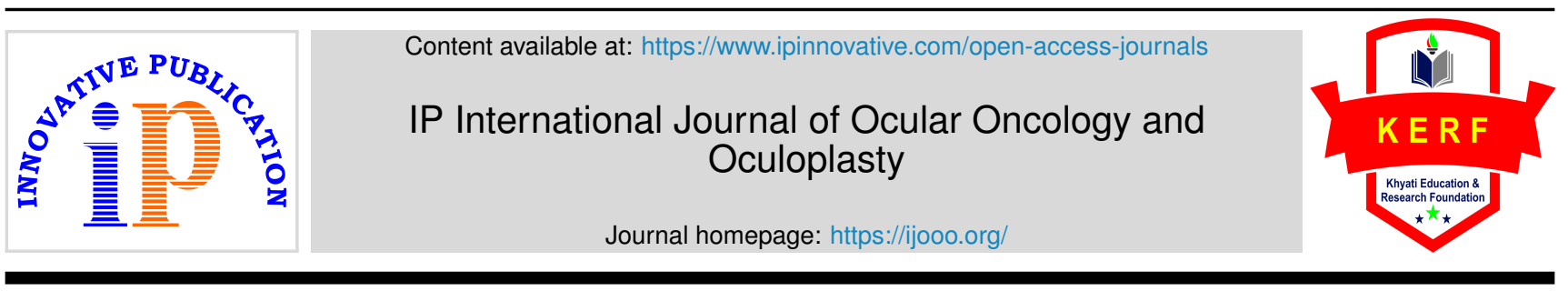

Original Research Article

\title{
Prevalence of dry eyes in patients with type 2 diabetes mellitus having meibomian gland dysfunction
}

\author{
Rani Sujatha M A ${ }^{\mathbf{1}}$, Sannidhi BJ ${ }^{\oplus 1}$,* \\ ${ }^{1}$ Dept. of Ophthalmology, Dr. B.R. Ambedkar Medical College, Bengaluru, Karnataka, India
}

\section{A R T I C L E I N F O}

\section{Article history:}

Received 12-03-2021

Accepted 16-03-2021

Available online 28-04-2021

\section{Keywords:}

Dry eye

Meibomian gland dysfunction

\begin{abstract}
A B S T R A C T
Purpose : The purpose of this study was to know the prevalence of dry eyes in patients with Type 2 Diabetes mellitus having meibomian gland dysfunction.

Materials and Methods : The study was conducted in patients visiting tertiary care hospital. This study included a total of 200 patients presenting with ocular discomfort, with history of Type 2 Diabetes mellitus. The patients were assessed for Meibomian gland dysfunction by noting the symptoms under slit lamp examination. Dry eyes were suspected on the basis of history including ocular discomfort, redness, blurred vision, gritty eyes and other sensation that improved with blinking. The examination for the dry eye was done using schirmer's test and tear break up time.

Results : A total of 130 out of 200 enrolled patients had meibomian gland dysfunction. The most common symptom being dryness $(55 \%)$, foreign body sensation(30\%), redness $(10 \%)$, gritty sensation(5\%).

Conclusion : The above study shows that the incidence of dry eyes is more in patients with Type 2 diabetes mellitus having Meibomian gland dysfunction.

Meibomian glands are important contributors for maintenance of healthy ocular surface. Meibomian gland dysfunction can cause qualitative and quantitative alteration in meibum with negative impact on the ocular surface.

(C) This is an open access article distributed under the terms of the Creative Commons Attribution License (https://creativecommons.org/licenses/by/4.0/) which permits unrestricted use, distribution, and reproduction in any medium, provided the original author and source are credited.
\end{abstract}

\section{Introduction}

Diabetes mellitus has been identified as one of the leading systemic risk factors in dry eye syndrome. ${ }^{1}$ Other common risk factor is female of post-menopausal age. ${ }^{2}$

Meibomian gland dysfunction is characterized by terminal duct obstruction and/or quantitative and qualitative changes in glandular secretion which leads to increased tear evaporation, thus causing dry eye. This is one of the leading causes of dry eye. ${ }^{3}$ Insulin and sex hormone plays important role in maintenance of normal meibomian glad function. ${ }^{2}$

\section{Materials and Methods}

A retrospective study was carried out in a total of 200 patients with ocular discomfort, with history of Type

\footnotetext{
* Corresponding author.

E-mail address: sannidhi.21@gmail.com (Sannidhi BJ).
}

2 diabetes mellitus attending the OPD, Department of ophthalmology in Dr. B.R. Ambedkar medical college over a period of 1 year.

The diabetic state was determined either by history of medication or abnormal random blood sugar $>200 \mathrm{mg} / \mathrm{dl}$ or HBA $1 \mathrm{C}>6.5 \%$ or fasting blood sugar $>126 \mathrm{mg} / \mathrm{dl}$.

\subsection{Inclusion criteria}

The age range of the subjects were between $40-65$ years.

\subsection{Exclusion criteria}

1. Patients with thyroid eye disease

2. Patients on medications like antihistamines and antidepressants.

3. Post cataract surgery (within 6 months) 
The patients were assessed for Meibomian gland dysfunction under slit-lamp examination.

Meibomian gland dysfunction was assessed for volume and viscosity by expression of the meibomian duct.

Table 1: Meibomian gland expression scale for volume

$\begin{array}{ll}\text { Grade } & \text { Volume } \\ 1 & \text { Normal volume: Just covers orifices } \\ 2 & \text { Increased 2-3 times the normal volume } \\ 3 & \text { Increased more than } 3 \text { times the normal volume } \\ 4 & \text { Increased to } 10 \text { times the normal volume }\end{array}$

Table 2: Meibomian gland expression scale for viscosity

\begin{tabular}{ll}
\hline Grade & Viscosity \\
1 & Normal, Clear, may have few particles \\
2 & Opaque with normal viscosity \\
3 & Opaque with Increased viscosity \\
4 & Severe thickening (tooth paste like) \\
\hline
\end{tabular}

The examination for the dry eye was done using schirmer's test and tear break up time

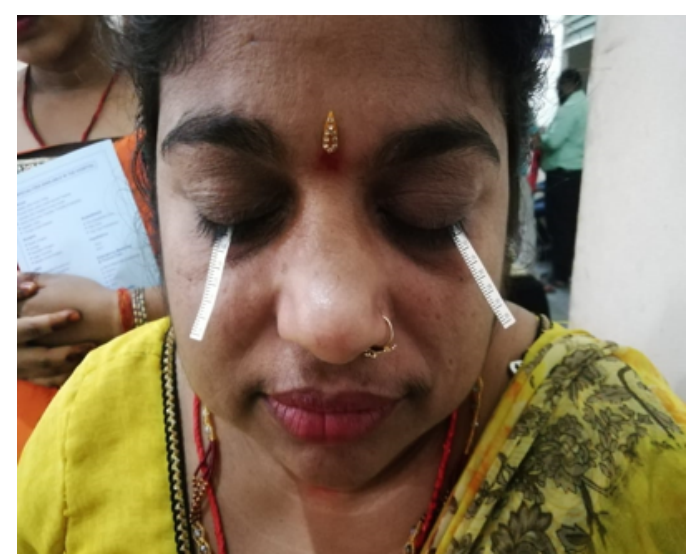

Fig. 1: Schirmer's test

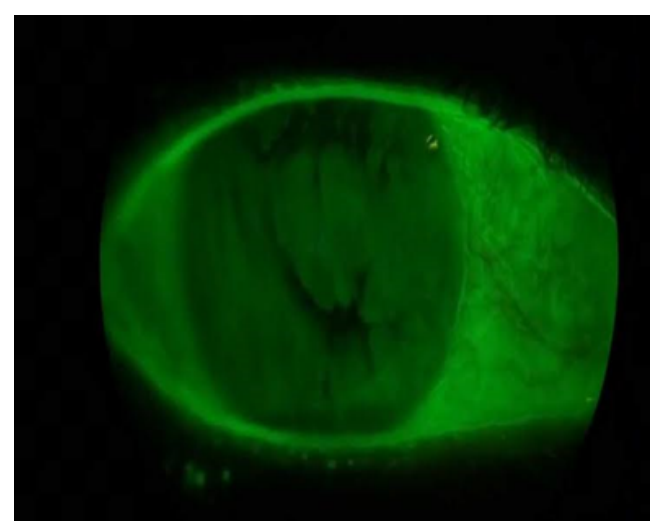

Fig. 2: Tear break up time

\subsection{Statistical analysis}

Mean, median, standard deviation, ranges were evaluated for continuous variables and for categorical variables, frequency and percentages were recorded. Chi-square test and ANOVA test were also used whenever necessary Independent $\mathrm{t}$ test was used to compare mean between the two group. $\mathrm{P}$ value of less than 0.05 within $95 \%$ CI was considered statistically significant

\section{Results}

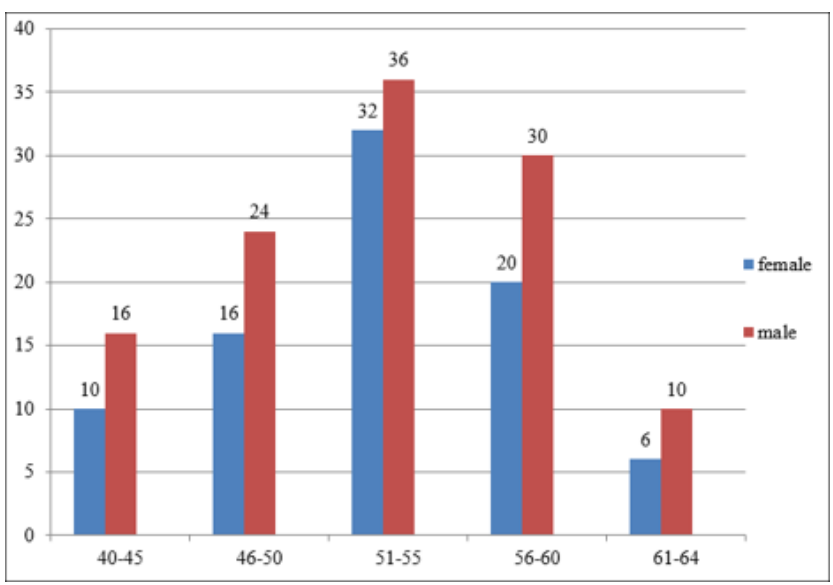

Fig. 3: Age and Gender distribution in diabetics

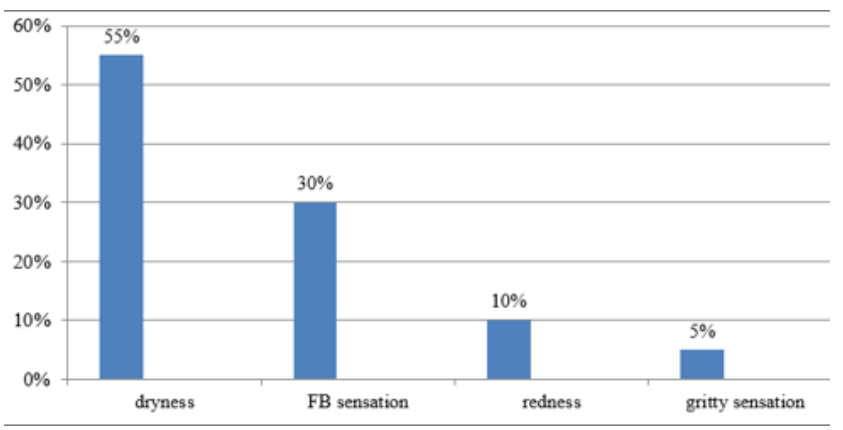

Fig. 4: Symptoms

Table 3: Meibomian gland expression scale for volume/viscosity

\begin{tabular}{lc}
\hline Grade & Diabetics (\%) \\
Normal & $168(84 \%)$ \\
Abnormal & $32(16 \%)$ \\
Total & $200(100 \%)$ \\
\hline
\end{tabular}

\section{Discussion}

1. Studies by Seifart and Strempel concluded that $70 \%$ of type 2 diabetics have proven dry eye disease. They also 
found that higher HBA1C values, greater the severity of dry eye disease. ${ }^{4}$

2. Ding et al. reported that in diabetic patients, hyperglycemia is one of the pathogenic factors causing common cause of dry eye. ${ }^{5}$

3. A study conducted by R.P. Shamsheer and Cynthia Arunachalam shows that the Meibomian gland dysfunction is an important cause of dry eye. This may be one of the causes for increased prevalence of dry eye in Diabetics. ${ }^{3}$

4. A study by Dr Jitendra kumar, Dr Preeti chaubey and Dr vijay Pratap concluded that the prevalence of Meibomian gland dysfunction in diabetic population was $56 \%$, MGD is an important pre disposer for Dry eye. $^{6}$

5. A study by Li et al, ${ }^{7}$ Kumar et al, ${ }^{6}$ Rathnakumar et al ${ }^{8}$ reported that the symptoms of MGD in type 2 Diabetes were highly significant especially burning and dryness.

6. A study by Igor Kaiserman MD, ${ }^{9}$ Sandrajohnna $\mathrm{G}^{10}$ and D.A Schaumberg ${ }^{11}$ showed that patients with MGD shows meibum expressibility abnormality, found more significantly in patients with type 2 diabetes mellitus.

\section{Conclusion}

1. Our study revealed high correlation between type 2 diabetes mellitus and meibomian gland dysfunction with dry eye.

2. Meibomian gland dysfunction is an important cause of dry eye and the frequency of the more severe form is greater in diabetics.

3. This may be one of the causes for the increased prevalence of dry eye in diabetics.

4. It should be noted at an early stage and treated appropriately in order to prevent more severe eye complications.

5. Therefore, examination of the meibomian gland is necessary, especially in long-term cases of diabetes mellitus.

\section{Conflicts of Interest}

All contributing authors declare no conflicts of interest.

\section{Source of Funding}

None.

\section{References}

1. Lin X. Meibomian Gland Dysfunction in Type 2 Diabetic Patients. $J$ Ophthalmol. 2017;2017:3047867. 100:10.155/2017/3047867.

2. Maurya RP, Singh VP, Chaudhary S, Roy M, Srivastav T. Prevalence of severe dry eye disease in postmenopausal women in North India: A teaching hospital study. Ind J Obs Gyne Res . 2019;6(1):94-6. त01:10.18231/2394-2/542010002]

3. Arunachalam C, Shamsheer RP. A clinical study of meibomian gland dysfunction in patients with diabetes. Middle East Afr J Ophthalmol. 2015;22(4):462-6. 001:10.4103/09/4-9233.16/827.

4. Seifart U, Strempel I. The dry eye syndrome and diabetes mellitus. Ophthalmologe. 1994;91(2):235-9.

5. Ding J, Liu Y, Sullivan DA. Effects of Insulin and High Glucose on Human Meibomian Gland Epithelial Cells. Invest Ophthalmol Vis Sci. 2015;56(13):7814-20. 10i:10. 16 Whiovs. 5-18049.

6. Kumar J, Chaubey P, Pratap V. A Clinical Study of Meibomian Gland Dysfunction in Patients with Diabetes in Bundelkhand region. IOSR J Dent Med Sci. 2017;16(06):14-8. 101-109700/0853-16060414/8

7. Li H, Pang G, Xu Z. Tear film function of patients with type two diabetes. Acta Academiae Med Sinicae. 2005;26(6):682-6.

8. Rathnakumar K, Ramachandran K, Ramesh V. Prevalence of dry eye disease in type 2 diabetic patients and its association with retinopathy. Int J Pharm Sci Res. 2017;8(10):4298-304.

9. Kaiserman I, Kaiserman N, Nakar S, Vinker S. Dry eye in diabetic patients. Am J Ophthalmol. 2005;139(3):498-503. do1:10.1016/].aj0.2004.10.022

10. Sandrajohanna G, Antonio L, Andrea G. Correlation between type 2 diabetes, dry eye \& MGD. J Optom. 2019;294:1-7.

11. Schaumberg DA, Nichols JJ, Papas EB, Tong L, Uchino M, Nichols KK, et al. The International Workshop on Meibomian Gland Dysfunction: Report of the Subcommittee on the Epidemiology of, and Associated Risk Factors for, MGD. Invest Ophthalmol Vis Sci.

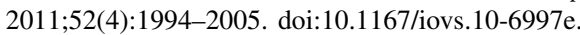

\section{Author biography}

Rani Sujatha M A, HOD

Sannidhi BJ, Post Graduate Student (1) https://orcid.org/0000-0003-49290224

Cite this article: Sujatha M A R, Sannidhi BJ. Prevalence of dry eyes in patients with type 2 diabetes mellitus having meibomian gland dysfunction. IP Int J Ocul Oncol Oculoplasty 2021;7(1):68-70. 